\title{
Effects of non-uniform strains on tensile fracture of fiber-reinforced ceramic composites
}

\author{
Varun P. Rajan, Frank W. Zok* \\ Materials Department, University of California, Santa Barbara, CA 93106, United States
}

\section{A R T I C L E I N F O}

\section{Article history:}

Received 1 February 2012

Received in revised form

11 July 2012

Accepted 17 July 2012

Available online 31 July 2012

\section{Keywords:}

Fracture

Fiber-reinforced composite material

Constitutive behavior

\begin{abstract}
A B S T R A C T
Effects of non-uniform strains on tensile fracture of fiber-reinforced ceramic-matrix composites have not been satisfactorily explained by existing mechanics-based models. In this paper, we use an exact model of fiber fragmentation under global load sharing conditions to predict fracture in three model problems in which non-uniform strains occur: (i) an end-constrained plate subject to a linear transverse temperature gradient; (ii) an internally-pressurized cylindrical tube with a linear through-thickness temperature gradient; and (iii) a rectangular beam under combined bending and tension. Fracture is assumed to occur when the global load reaches a maximum value. Approximations to the exact fragmentation model are also assessed, with the goal of decoupling the effects of two important parts of the computed stress-strain response: the rate of post-peak strain softening and the magnitude of the plateau "flow" stress once fiber fragmentation is complete. We find that for cases in which the fiber Weibull modulus is low and hence its plateau strength is high relative to its peak and the loading yields a sufficiently high strain gradient, the failure strain lies in the plateau regime. Consequently, the results can be predicted with good accuracy using a perfectly-plastic representation of the post-peak response. In contrast, for cases in which the fiber Weibull modulus is high, the failure strain lies in the softening portion of the curve. Here a linear-softening model is found to yield accurate results. A preliminary assessment of the model has been made by comparing predicted and measured bending/tension strength and failure strain ratios for one specific composite. The correlations appear good, though additional experiments are required in order to critically assess the model predictions over a range of loading scenarios.
\end{abstract}

(c) 2012 Elsevier Ltd. All rights reserved.

\section{Introduction}

Fracture of tough fiber-reinforced ceramic-matrix composites (CMCs) occurs through a process of multiple matrix cracking followed by fiber fragmentation and subsequent pullout of broken fiber fragments. Through this process, the average stress borne by the matrix quickly diminishes with increasing strain and the majority of the load is eventually supported by the fibers. Consequently, the composite strength is dictated by the fiber bundle strength. Numerous studies over the past two decades have addressed the mechanics and statistics of fiber fragmentation and the corresponding ultimate tensile strength and strain (Curtin, 1991a, b; Curtin et al., 1998; Hui et al., 1995; Neumeister, 1993a; Phoenix, 1993; Thouless and Evans, 1988). A review article by Curtin (1998) summarizes many of the important developments.

\footnotetext{
* Corresponding author. Tel.: +1 805893 8699; fax: +1 8058938486 .

E-mail address: zok@engineering.ucsb.edu (F.W. Zok).
} 
Most of the studies have been based on a one-dimensional shear lag analysis to describe the stress build-up around broken fibers, assuming that the frictional resistance between the fibers and matrix is constant within the region in which the fibers have slipped past the matrix. Furthermore, most have been based on the assumption of global load sharing; that is, the load carried previously by a broken fiber is shed equally to all other fibers in the plane of the break without generating stress concentrations in neighboring fibers. Notable exceptions to the latter include the work of Ibnabdeljalil and Curtin (1997).

One of the outstanding challenges in the mechanics of composite fracture pertains to the effects of non-uniform strains. The latter arise from bending moments or constrained thermal expansion in non-uniform temperature fields. They also occur around discontinuities such as notches and holes. The experimental evidence suggests that there are two distinct effects of non-uniform strains.

First, inelastic strain associated with matrix cracking and fiber fragmentation leads to stress re-distribution (Cady et al., 1995; McNulty et al., 1999). In the case of bending of a rectangular beam, for instance, inelasticity leads to a non-linear stress distribution and a progressive shift in the neutral axis from the beam center towards the compressive face. Even making the conservative assumption that failure occurs upon attainment of a critical tensile strain, the predicted nominal bending strength (calculated on the basis of elastic analysis) exceeds the uniaxial tensile strength by a large margin (Beyerle et al., 1992; Marshall and Evans, 1985; McNulty and Zok, 1997). Indeed, experimental measurements show that the bending strengths of typical CMCs are about 50-120\% greater than their respective tensile strengths (Beyerle et al., 1992; Marshall and Evans, 1985; McNulty and Zok, 1997; Prewo, 1986).

A second effect (not broadly recognized) is manifested as a disparity between the peak tensile strain attained in pure bending and the failure strain in uniaxial tension. Typical values for one CMC are shown in Table 1 (McNulty and Zok, 1997); here the failure strain in bending is about 50\% greater than that in tension. Yet higher values of tensile strains have been measured in the vicinity of notches or holes in tensile coupons of the same CMC (McNulty et al., 1999). Similar trends have been reported for fiber-reinforced metal-matrix composites. For instance, in SiC-fiber titanium-matrix systems, the peak tensile strains attained in bending exceed that the uniaxial tensile fracture strain by more than $60 \%$ (Ramamurty, 2004). These results demonstrate that the tensile strain for fracture of fiber-reinforced composites is not unique and thus models based on a critical strain criterion are likely to underestimate their full potential.

Hild et al. (1994) attempted to predict fracture of CMCs in bending using a constitutive law derived from fiber fragmentation theory coupled with a critical tensile strain criterion for fracture. The model was capable of capturing some of the effects of inelasticity on stress distribution but, clearly, could not predict the differing failure strains in bending and tension. Neumeister (1993b) used a similar approach to address the same problem but with an alternate (ad hoc) fracture criterion: notably, one based on the attainment of a critical number of fiber breaks within a prescribed volume element. In addition to lacking a sound fundamental basis, the fracture criterion leads to a predicted tensile-face failure strain in bending comparable to the failure strain in tension: again inconsistent with experimental measurements (McNulty and Zok, 1997). Yet others have attempted to rationalize the effects of non-uniform strains on the basis of weakest link scaling laws, wherein strength increases as the volume of stressed material decreases (Bullock, 1974; McNulty and Zok, 1997; Whitney and Knight, 1980). Although this approach has been made to bring some experimental results into agreement with the model - by tuning the parameters in an assumed Weibull distribution, for instance - it is purely phenomenological and lacks predictive power.

Steif and Trojnacki (1994) presented an analysis of the bending problem that was based on a purely phenomenological representation of the tensile response of a composite (without any explicit connection to fiber properties) but proved to be useful in demonstrating the role of the post-peak strain-softening rate on the composite bending strength. To this end, they used a one-dimensional tri-linear constitutive law for the composite. The three parts were intended to depict: (i) the linear-elastic portion before matrix cracking in tension as well as that in compression; (ii) the reduction in tangent modulus due to matrix cracking; and (iii) the strain softening beyond the peak stress. They then used standard EulerBernoulli beam theory coupled with a fracture criterion based on the attainment of a maximum bending moment to compute the nominal bending strength. They concluded that the degree to which the bending strength exceeds the tensile strength is governed predominantly by the rate of post-peak strain softening. In the present paper, we show, using a similar analysis but with a mechanistic model for fiber fragmentation, that there are indeed circumstances in which the bending/ tension strength differential can be attributed to the post-peak softening. We also show that there are other circumstances in which this is not the case; instead, the dominant effect is associated with the attainment of a plateau flow stress after fiber fragmentation is complete (a feature not captured by the model of Steif and Trojnacki). We also examine the effects of strain softening and plateau flow on failure in other loading scenarios in which strain gradients are present.

Table 1

Measured failure strains and stresses in uniaxial tension, four-point bending, and open-hole tension of a laminated $\left[0^{\circ} / 90^{\circ}\right]$ laminated Nicalon/MAS composite (McNulty and Zok, 1997).

\begin{tabular}{lll}
\hline Test & $\begin{array}{l}\text { Maximum local strain } \\
\text { at failure (\%) }\end{array}$ & Strength (MPa) \\
\hline Uniaxial tension & $0.86-0.93$ & $299-324$ \\
Four-point bending & $1.30-1.40$ & $600-650$ (flexural) \\
Open-hole tension & 1.60 & - \\
\hline
\end{tabular}


An argument could be made that, once the stress in a composite structure locally reaches the peak in its tensile stressstrain curve, the deformation would localize into a narrow band and thus the assumption of deformation homogeneity implicit to the load-maximum failure criterion would break down. If this were the case, the preferred approach to failure prediction would involve use of a cohesive zone model. We present arguments later in the paper that, if the localization spreads under a progressively increasing macroscopic load (i.e. with structural hardening), then other potential localization sites remote from the first can be triggered, leading to an array of such bands, with a characteristic spacing controlled by the fragment length. Thus, although the deformation would not be homogeneous over distances comparable to the crack spacing, the displacements could be averaged over an appropriate length scale so as to compute a physicallymeaningful strain.

Assessments of the fidelity of predicted bending strengths have been based on comparisons between such predictions and experimental measurements. However, some of these assessments are tainted by the fact that failure in the experiments had not occurred in a purely tensile mode. For example, in the studies of Marshall and Evans (1985), Prewo (1986), and Jansson and Leckie (1992) on unidirectional SiC/LAS, tensile fracture was preceded by compressive crushing at the loading points in the bend tests. In another study, by Beyerle et al. (1992) on unidirectional SiC/CAS, fracture in bending occurred via delamination in the regions between the inner and outer loading points: not in the region of maximum moment. Consequently, conclusions drawn from such comparisons (Hild et al., 1994; Neumeister, 1993b; Steif and Trojnacki, 1994) should be judged skeptically. (It is noted parenthetically that, because of the very high tensile strength of unidirectional CMCs coupled with their low resistance to in-plane shear loads, true tensile failure is generally difficult to attain under flexural loading. The problem is less pronounced in multidirectional laminates.)

The principal objective of the present study is to develop a mechanics framework to describe the effects of non-uniform strains on tensile fracture of fiber-reinforced CMCs under monotonic loading. The outline of the paper is as follows. We begin with a description of the constitutive law for the composite tensile stress-strain response, based on the fiber fragmentation model of Hui et al. (1995). Next, we present a framework that utilizes this constitutive law to ascertain the maximum load-bearing capacity of composite structures in the presence of non-uniform strains. The key assumption (as noted earlier) is that failure occurs upon attainment of a global load maximum. This framework is then applied to three model problems of engineering interest: (i) an end-constrained plate subjected to a linear transverse temperature gradient; (ii) a cylindrical thin-walled tube with a linear through-thickness temperature gradient and internal pressure; and (iii) a beam with rectangular cross-section undergoing combined bending and tension. Each problem produces a constant strain gradient in one of the directions transverse to the fibers. We find that the peak tensile strain at the load maximum increases with the magnitude of the strain gradient; the sensitivity of the failure strain to the strain gradient is not unique but rather depends on the loading path. Furthermore, in order to glean insights into the characteristics of the tensile response that control failure, we consider approximate representations that decouple the effects of strain softening from the plateau flow stress at large strains. Finally, calculations of failure strain and stress for the problem of pure bending are assessed against the experimental data of McNulty and Zok (1997).

\section{Constitutive laws}

\subsection{Fiber fragmentation}

The inelasticity in the post matrix-cracking tensile response is governed by the process of fiber fragmentation and sliding along the fiber-matrix interfaces. A convenient description of the statistical variation in fiber strength is the twoparameter Weibull distribution:

$$
F=1-\exp \left[\frac{-L}{L_{0}}\left(\frac{\sigma_{f}}{\sigma_{0}}\right)^{\rho}\right]
$$

where $F$ is the cumulative failure probability of a fiber of length $L$ at a stress $\sigma_{f}, L_{0}$ and $\sigma_{0}$ are reference values of length and strength, respectively, and $\rho$ is the Weibull modulus. Shear-lag theory describes how the fiber stress away from a break is restored to its far-field value, via the frictional resistance $\tau_{0}$ of the fiber-matrix interface (Cox, 1952). Assuming that $\tau_{0}$ is constant, the fiber stress varies linearly within a "recovery zone" adjacent to the fiber break, over a length $l=2 R \sigma_{f} / \tau_{o}$ where $\sigma_{f}$ is the fiber stress remote from the break, given by $\sigma_{f}=E_{f} \varepsilon$, with $E_{f}$ being the fiber modulus and $\varepsilon$ the applied strain.

The fiber fragmentation problem has been analyzed approximately by Curtin (1991a, b) and Neumeister (1993a) and solved exactly by Hui et al. (1995). The differences in the resulting constitutive laws from the exact and the approximate analyses arise from differing assumptions about two physical phenomena: shadowing of fiber defects within the recovery zone and overlap of recovery zones adjacent to neighboring breaks (Neumeister, 1993a). Neglecting these phenomena leads to erroneous predictions in the post-peak softening regime (Neumeister, 1993a). The constitutive law employed here is the one based on Hui's solution. The model is based on the assumptions that: (i) the frictional resistance of the fiber/ matrix interface is uniform along the length of the fibers (unaffected by local perturbations associated with discrete matrix cracks); (ii) the characteristic terminal fragment length is small compared to the gauge length of stressed material; and (iii) the fiber strength follows a Weibull distribution (Eq. (1)). Suitable normalizations yield three key parameters: (i) a non-dimensional fiber stress, $S=\bar{\sigma} / V_{f} \sigma_{c}$ where $\bar{\sigma}$ is the average stress borne by the fibers, $V_{f}$ is the volume fraction of fibers aligned with the tensile loading direction and $\sigma_{c}$ is a characteristic strength defined by $\sigma_{c}=\left(\sigma_{0}^{\rho} \tau_{0} L_{0} / R\right)^{1 /(\rho+1)}$; (ii) the 


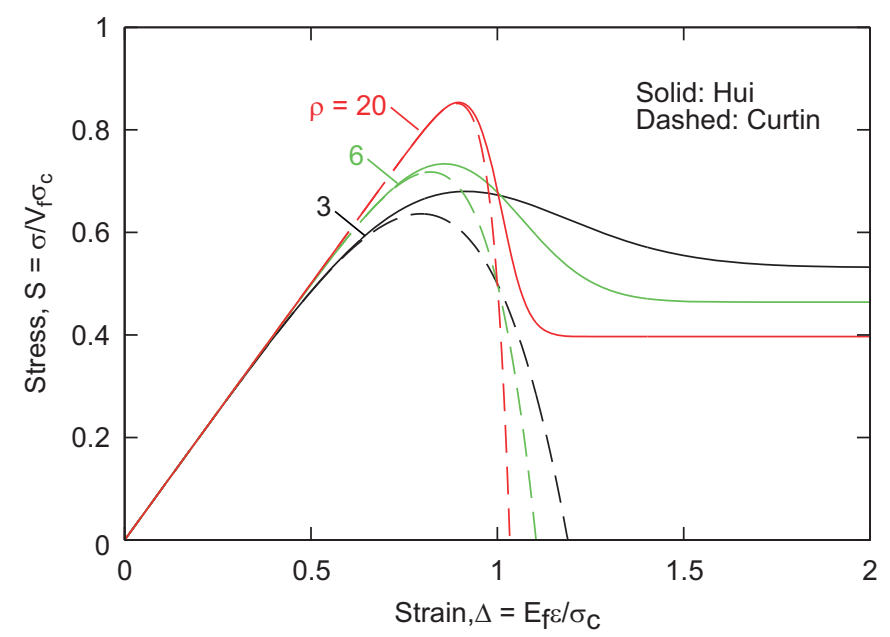

Fig. 1. Comparison of constitutive laws for three typical values of $\rho$.

corresponding tensile strain, $\Delta=\varepsilon E_{f} / \sigma_{c}$; and (iii) a characteristic length, given by $\Delta_{c}=\left(R \sigma_{0} / \tau_{0} L_{0}^{1 / \rho}\right)^{\rho /(\rho+1)}$. The full analytical description of the stress-strain response is presented in the Appendix A. The integrals contained therein were computed numerically in MATLAB (Mathworks, 2009) using adaptive Simpson quadrature.

Tensile stress-strain curves computed from Hui's model for representative values of $\rho$ are depicted in Fig. 1. The notable features include the gradual strain softening following the peak and a "plateau" flow stress at large strains (once the fragmentation process is complete). Also shown for comparison are the stress-strain curves from Curtin's approximate model. The latter yields reasonably accurate results up to the peak stress but not in the post-peak (softening) regime.

\subsection{Complete constitutive law}

To ensure that the composite is modeled in a physically-realistic manner in the elastic regime as well as under compressive loading, the preceding fragmentation model is supplemented with a pre-matrix cracking constitutive law. The compressive response of the composite is taken to be elastic for all strains, and the elastic moduli in compression and tension are assumed to be equal to one another. The ratio $E^{\prime}$ of the post-matrix cracking tangent modulus to the longitudinal composite modulus $E_{c}$ is defined by $E^{\prime}=V_{f} E_{f} / E_{c}$. For typical fiber volume fractions and fiber/matrix moduli, $E^{\prime}$ falls between $1 / 4$ and $1 / 2$. In addition, although matrix cracking is experimentally observed to commence and saturate at somewhat different stress levels (Evans and Zok, 1994), the constitutive law used in the present analysis assumes that these stresses are the same. The consequence is that the stress-strain curve exhibits a strain burst upon matrix cracking, in accord with the prediction of the well-known micromechanical model of Aveston et al. (1971). A non-dimensional matrix cracking stress is defined by $S_{m c}=\sigma_{m c} / V_{f} \sigma_{c}$. The complete constitutive law can thus be expressed in piecewise form as

$$
S(\Delta)= \begin{cases}\Delta / E^{\prime} & \Delta<S_{m c} E^{\prime} \\ S_{m c} & S_{m c} E^{\prime}<\Delta<S_{m c} \\ f(\Delta) & S_{m c}<\Delta\end{cases}
$$

where $f(\Delta)$ is the fiber bundle response presented in the Appendix A.

In the model problems we consider in the present article, the structures do not undergo unloading either globally or locally. Consequently, differences in the loading/unloading response (common in CMCs) are neglected.

\subsection{Approximate representations}

In order to glean insights into the important post-peak characteristics that govern failure, we also consider two approximate formulations of the constitutive law obtained from Hui's model (Fig. 3). In the first, termed the plateau model, the post-peak response is represented by instantaneous softening from the peak to the plateau and the subsequent flow stress is assumed to remain at the plateau level for higher strains. This representation neglects the strain-softening portion of the curve and is expected to yield a lower bound on the predicted failure stress and strain. In the second, termed the softening model, the post-peak response is assumed to follow a linear softening law, with the rate of softening obtained from a linear fit of the exact results in the regime between the peak and the plateau (Fig. 3). The expectation is that, for small strain gradients, failure will occur at strains only slightly beyond the peak and hence the linear softening model should yield reasonably accurate results. Conversely, for large gradients, the failure strain will fall well within the plateau 


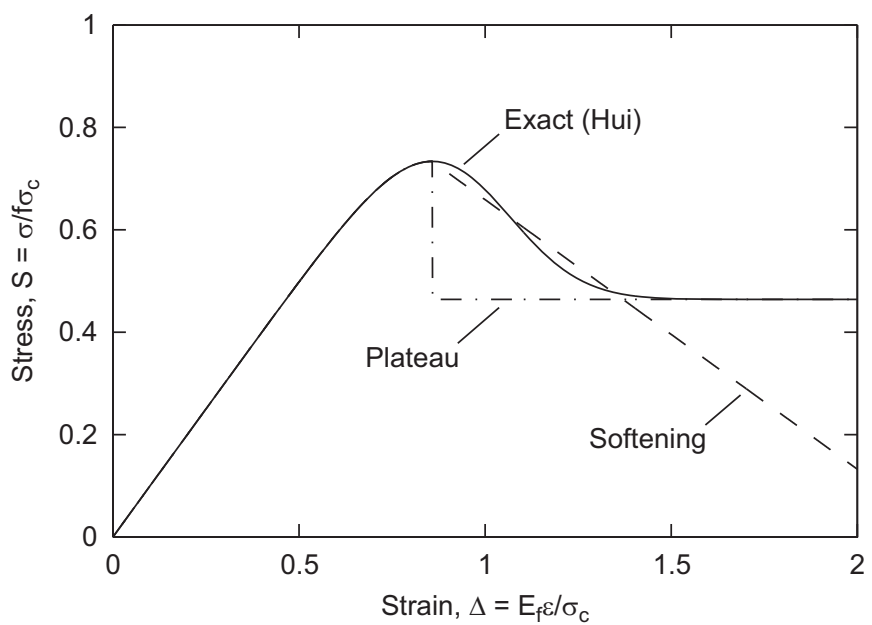

Fig. 2. Two approximate representations of the post-peak response: perfectly-plastic flow at the plateau stress and linear softening.

a
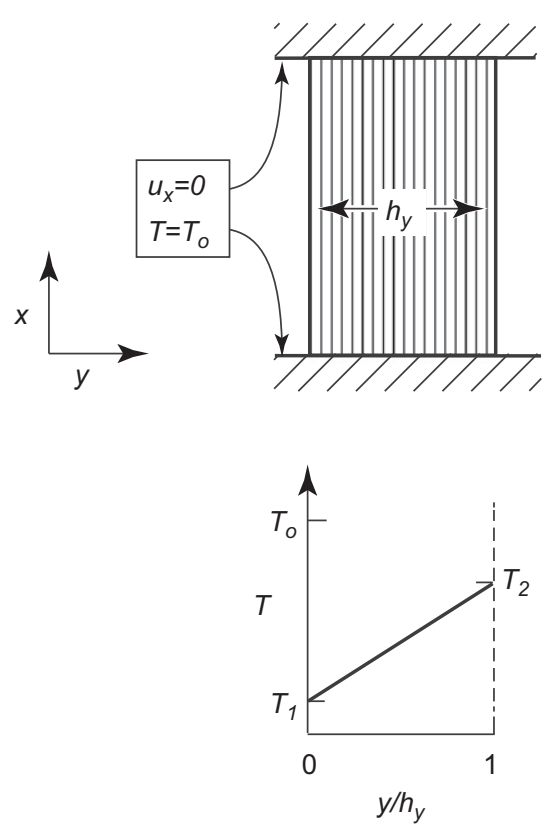

b
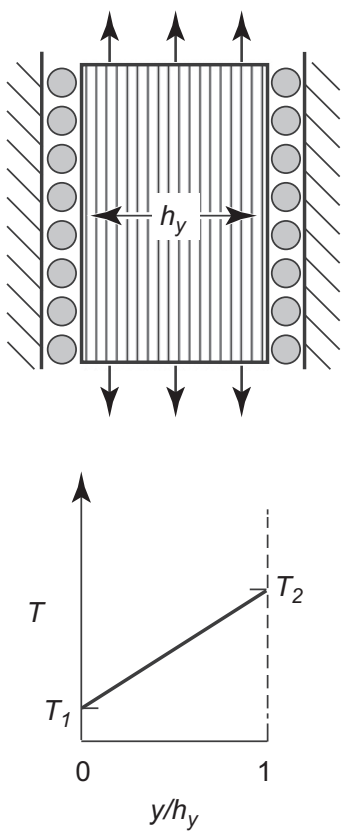
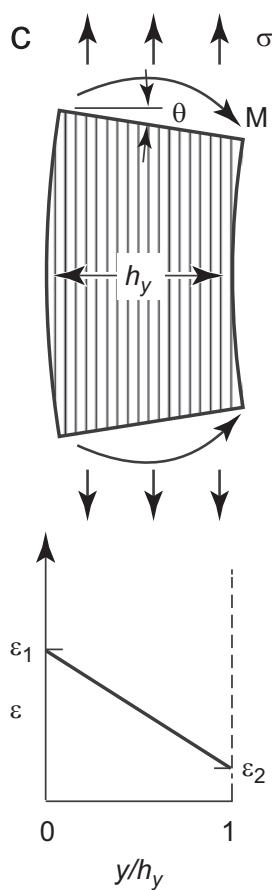

Fig. 3. Schematics of the three model problems of interest: (a) linear temperature gradient across an end-clamped plate, (b) pressurized tube with linear temperature gradient through the wall, and (c) combined bending and tension of a rectangular beam.

regime, and thus the perfectly-plastic post-peak representation should yield more accurate results. A further expectation is that the suitability of the two approximate representations will depend on $\rho$. This is because, as $\rho$ decreases, the ratio of the plateau stress to the peak stress increases and the rate of strain softening decreases.

Assessments of the two approximate representations are made on the basis of the errors in the predicted strengths of the composite structures relative to those obtained from the exact Hui model. The results are presented as maps in coordinates of fiber Weibull modulus and a measure of the strain gradient. The maps depict the regimes in which the approximate models yield sufficiently accurate results: an error of less than 5\% being deemed to be adequate. For comparison, a model based on the attainment of a critical fracture strain is also considered. Consequently, in general, three (potentially overlapping) domains emerge, one for each of the three approximate representations. A fourth domain - one in which none of the three approximations provide adequate accuracy - can also be present. 


\section{Model problems}

\subsection{Approach}

The approach for solving the problem of fracture with non-uniform strains is as follows. Eq. (2) defines a constitutive law that relates normal strains and stresses in the fiber direction, $x$. The axial strain is permitted to vary only in the transverse directions, $y$ and $z$. The reaction force, $r_{x}$, in the fiber direction can therefore be expressed as

$$
r_{x}=\int_{A} \sigma_{x x}\left(\varepsilon_{x x}(y, z)\right) \mathrm{d} y \mathrm{~d} z
$$

The $y$ and $z$ coordinates are non-dimensionalized as $Y=y / h_{y}$ and $Z=z / h_{z}$, where $h_{i}$ is the dimension of the specimen in the $i$ direction. Then, utilizing the normalizations introduced in Section 2, Eq. (3) reduces to

$$
R_{x}=\frac{r_{x}}{h_{y} h_{z} V_{f} \sigma_{c}}=\int_{A} S(\Delta(Y, Z)) \mathrm{d} Y \mathrm{~d} Z
$$

The strain distribution, $\Delta(Y, Z)$, is assumed to be known a priori. The loading trajectory is expressed in terms of a single parameter, $t$ : for instance, an applied displacement, a rotation or a temperature gradient. Fracture is deemed to have occurred when the reaction force attains a maximum (not when a material element locally reaches the peak in its stressstrain curve). The fracture strength, $S^{f}$, is thus obtained by maximizing $R_{x}$ with respect to $t$. Similarly, the fracture strain, $\Delta_{\max }^{f}$, is taken as the maximum value of $\Delta(Y, Z)$ within the body when the critical value of $t$ is reached. Numerical solutions were obtained using a minimization algorithm in the optimization toolbox of MATLAB (Mathworks, 2009).

For problems involving bending, a non-dimensional moment is defined as

$$
M_{z}=\frac{m_{z}}{h_{y}^{2} h_{z} V_{f} \sigma_{c}}=\int_{A}\left(Y-Y_{t}\right) \cdot S(\Delta(Y, Z)) \mathrm{d} Y \mathrm{~d} Z
$$

where $Y_{t}$ is the $Y$-coordinate of the axis of applied tension. Proportional loading is assumed, with $M_{z} / R_{x} \equiv \tan (\lambda)$, where $\lambda$ characterizes the mixity of loading. The loading reduces to pure bending for $\lambda=\pi / 2$ and pure tension for $\lambda=0$. Intermediate values of $\lambda$ describe combined bending and tension. Numerical solutions were again obtained using MATLAB (Mathworks, 2009). Since the moment and the axial load are proportional, either quantity can be used as the objective function for maximization. The loading parameter was taken to be the tensile-face strain $\Delta_{\max }$ For each prescribed value of $\Delta_{\text {max }}$, a subroutine was used to solve numerically for the strain gradient, $\Psi$, that satisfies the proportional loading equation

$$
M_{z}\left(\Delta_{\max }, \Psi\right)=\tan (\lambda) \cdot R_{x}\left(\Delta_{\max }, \Psi\right)
$$

For pure bending, the quantities of interest obtained from the numerical solution are the tensile-face strain at failure, $\Delta_{\text {max }}^{f}$, and the failure moment, $M_{z}^{f}$. The flexural strength, $\sigma_{b}^{f}$, is expressed in non-dimensional form in accordance with

$$
S_{b}^{f}=\frac{\sigma_{b}^{f}}{V_{f} \sigma_{c}}=6 M_{z}^{f}
$$

where $S_{b}^{f}$ is the non-dimensional flexural strength. For combined bending/tension, a failure locus can be constructed at a constant value of $\rho$. The moment at failure, $M_{z}^{f}$, is converted into a flexural strength using Eq. (7), while the axial load at failure, $R_{x}^{f}$, becomes the tensile strength, $S_{t}^{f}$.

In each of the three model problems considered below, the strain distribution is linear and varies in only the $Y$ direction. The strain distribution can therefore be expressed as

$$
\Delta(Y, Z)=\Delta_{\max }-\Psi \cdot Y
$$

where $\Delta_{\max }$ is the maximum tensile strain; the origin of the $Y$-axis is positioned so that the maximum strain is located at $Y=0$; and $\Psi$ characterizes the strain gradient. Both $\Delta_{\max }$ and $\Psi$ are functions of the loading parameter $t$.

\subsection{Constrained plate with temperature gradient}

In the first model problem, we consider a composite plate with a rectangular cross-section fixed between two rigid supports and initially at a uniform (stress-free) temperature $T_{0}$ (Fig. 3(a)). The left side of the plate is positioned at $Y=0$ and its temperature, $T_{L}$, is reduced at a constant rate so that $T_{L}=T_{0}-\dot{T}_{L} \tau$, where $\tau$ is time. Similarly, the right side of the plate is cooled at a different rate in accordance with $T_{R}=T_{0}-\dot{T}_{R} \tau$. Without loss of generality, we assume that $\dot{T}_{L} \geq \dot{T}_{R}$. Further assuming that the temperature gradient thereby developed is linear, the thermal strain field becomes

$$
\varepsilon_{t h}(Y, \tau)=\alpha\left[T_{L}(1-Y)+T_{R}-T_{0}\right]=-\alpha \tau\left[\dot{T}_{L}(1-Y)+\dot{T}_{R}\right]
$$


where $\alpha$ is the coefficient of thermal expansion of the composite in the fiber direction. Since the total strain is zero, the mechanical strain is $\varepsilon_{m}=-\varepsilon_{t h}$. Its maximum value, written in the form of Eq. (8), is

$$
\Delta_{\max }(\tau)=\frac{E_{f} \alpha \dot{T}_{L} \tau}{\sigma_{c}}
$$

The corresponding strain gradient is

$$
\Psi(\tau)=\Theta \cdot \Delta_{\max }(\tau)
$$

where

$$
\Theta=1-\frac{\dot{T}_{R}}{\dot{T}_{L}}
$$

All physically-plausible scenarios fall in the domain $0 \leq \Theta \leq 1$.

Illustrative numerical results showing the effects of $\rho, S_{m c}$ and $E^{\prime}$ on the fracture strength and strain are presented in Figs. 4 and 5. They show that the fracture strain increases monotonically with strain gradient (characterized by $\Theta$ ). The effects are most pronounced for small Weibull moduli ( $\rho \leq 5$, typical of SiC fibers). The fracture stress, however, decreases
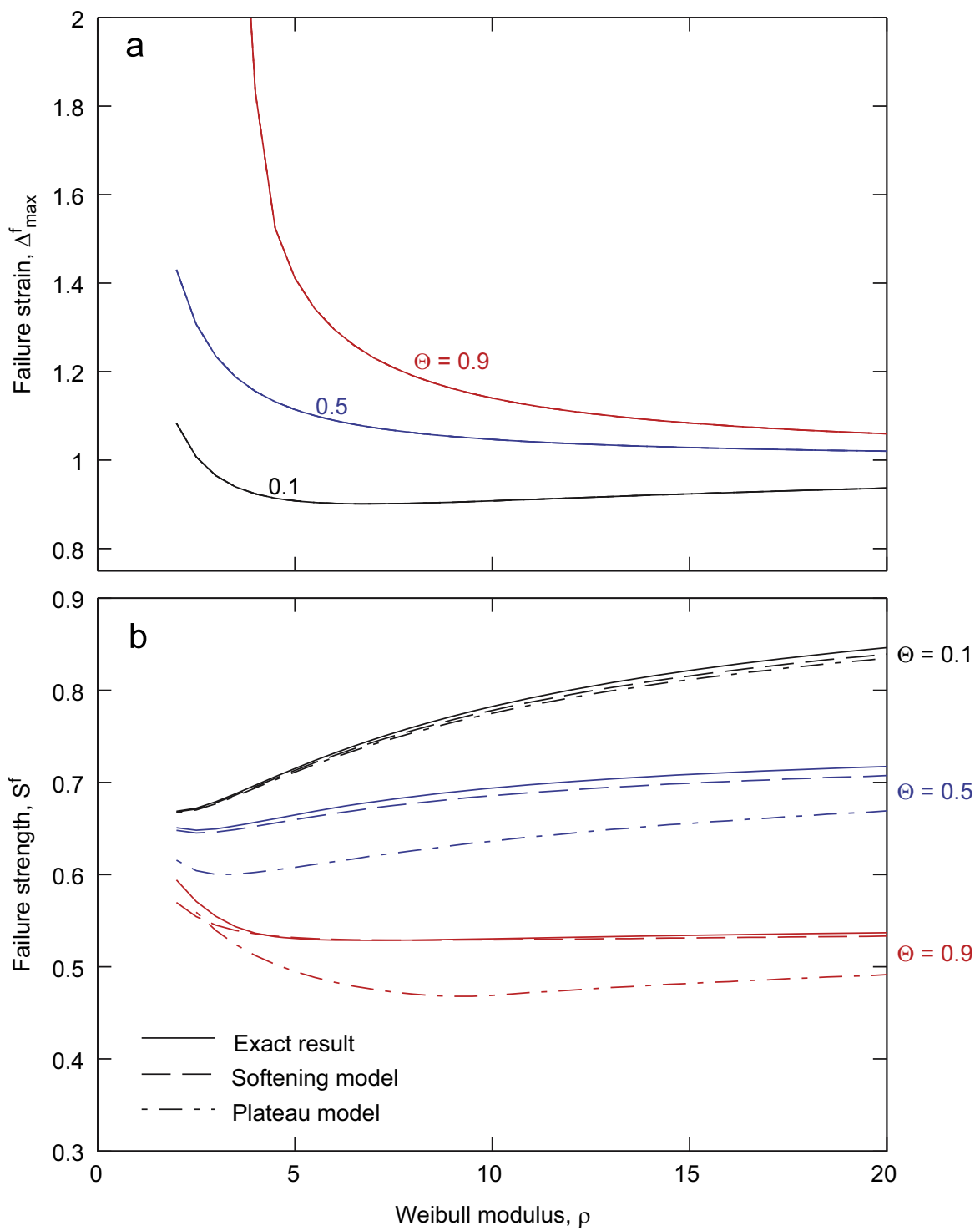

Fig. 4. Effects of Weibull modulus $\rho$ and strain gradient $\Theta$ on (a) the maximum strain at peak load and (b) the corresponding peak stress for the linear temperature gradient problem $\left(E^{\prime}=1 / 3\right.$ and $\left.S_{m c}=0\right)$. 

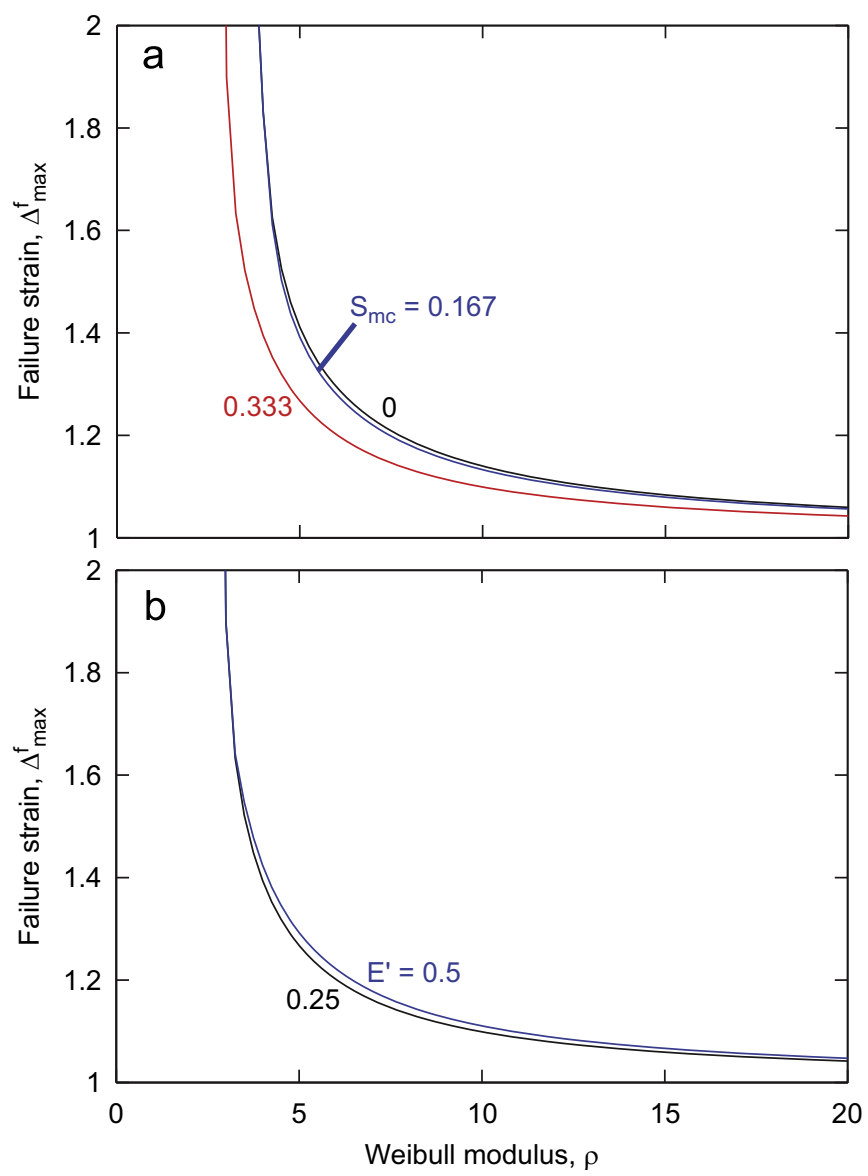

Fig. 5. Effects of (a) matrix cracking stress $S_{m c}$ (for $\Theta=0.9$ and $E^{\prime}=1 / 3$ ) and (b) the modulus ratio $E^{\prime}$ (for $\Theta=0.9$ and $S_{m c}=1 / 3$ ) on the maximum strain at the peak stress for the linear temperature gradient problem.

with $\Theta$. The results also indicate that the fracture stress and strain are insensitive to both $S_{m c}$ and $E^{\prime}$. This insensitivity arises because, at fracture, the majority of the material resides in the post-matrix-cracking regime.

Also shown for comparison in Fig. 4(b) are the predictions based on the linear softening model and the plateau model. Here the softening model yields accurate results over the entire range of $\rho$ and $\Theta$. The plateau model, in contrast, is adequate at low strain gradients but underestimates the strength for $\Theta \geq 0.3$.

A map showing the domains in which the approximate models yield accurate results is presented in Fig. 6 . It comprises three regimes. (i) For small gradients, characterized approximately by $\Theta \leq 1 / 3$, fracture occurs shortly after the peak in the stress-strain curve. Consequently, all three of the approximate models yield adequate results. The boundary of this domain is almost independent of $\rho$. (ii) The plateau model is also accurate in the domain of very low values of $\rho$ and high strain gradients. This result arises because the plateau stress is a significant fraction of the peak value at low values of $\rho$ and thus, under large strain gradients, failure occurs when the peak strain is well within the plateau domain. (iii) The linear softening model provides adequate results over the entire parameter space. The inference is that the additional loadbearing capacity (beyond that at the attainment of the peak stress locally) is attributable largely to the finite post-peak strain-softening rate; the plateau flow stress plays a decidedly secondary role.

\subsection{Pressurized tube with temperature gradient}

We next consider a thin-walled cylindrical tube subject, first, to a linear through-thickness temperature gradient and, second, to a monotonically increasing internal pressure. Fibers are oriented in the circumferential direction. The rotational symmetry of the problem precludes bending; however, a net mechanical stress must develop over the cross-section to counterbalance the internal pressure (Fig. 2(b)). Combining this equilibrium condition with the result in Eq. (4) yields the reaction force $R_{x}$

$$
R_{x}=\frac{p d}{2 h_{y} V_{f} \sigma_{c}}
$$




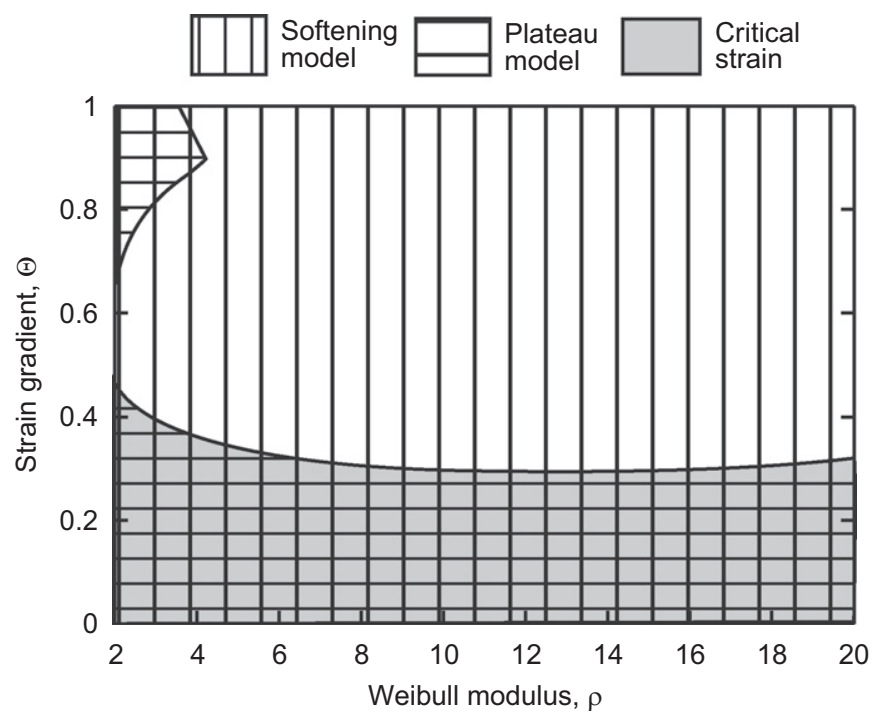

Fig. 6. Failure map for the linear temperature gradient problem $\left(E^{\prime}=1 / 3\right.$ and $\left.S_{m c}=0\right)$.

where $p$ is the internal pressure, $d$ is the diameter of the tube, and $h_{y}$ is the thickness of the wall. With the wall mid-plane held at the stress-free temperature, the thermal strain distribution is $\varepsilon_{t h}=\alpha \Delta T(Y-1 / 2)$ where $\Delta T$ is the through-thickness temperature difference and $Y=y / h_{y}$. The mechanical strain therefore becomes $\varepsilon_{\text {mech }}=\varepsilon_{\text {tot }}+\alpha \Delta T(1 / 2-Y)$, where the total strain is independent of $Y$ since the tube is thin-walled. Upon normalization, this expression can be rewritten in the form of Eq. (8), with

$$
\Psi=\frac{E_{f} \alpha \Delta T}{\sigma_{c}}
$$

and

$$
\Delta_{\max }=\Delta_{t o t}+\frac{\Psi}{2}=\frac{\varepsilon_{t o t} E_{f}}{\sigma_{c}}+\frac{\Psi}{2}
$$

Taking the temperature difference to be constant, the parameter describing the loading trajectory is $\Delta_{\max }$, which increases monotonically with pressure.

Select numerical results are plotted in Fig. 7. Here again the failure strain increases with the gradient (characterized in this case by $\Psi$ ), albeit with a significantly weaker dependence on $\rho$. Also, the failure stress and strain exhibit an even weaker dependence on $S_{m c}$ and $E^{\prime}$ (not shown) because only a small portion of the tube wall remains in the elastic domain at fracture.

The failure map for this problem is shown in Fig. 8. It exhibits characteristics similar to those seen in the previous problem. Notably, for low strain gradients, all three of the approximate models are adequate since failure occurs shortly after the peak stress is attained locally. The predictions of the plateau model and the critical strain model begin to break down at a critical strain gradient, characterized by $\Psi \leq 0.2$ (almost independent of $\rho$ ), while the softening model yields accurate results over most of the parameter space. However, the softening model also eventually breaks down, at very high gradients and high $\rho$. The increasing error in the predictions from the softening model with increasing $\rho$ are evident in Fig. 7 (b) for the case $\Psi=0.9$.

\subsection{Combined bending and tensile loading}

Finally, we consider a beam with rectangular cross-section subjected to proportional bending plus tension (Fig. 2(c)). From Euler-Bernoulli bending theory, the strain distribution is linear through the cross-section and can be written in the form of Eq. (8), with

$$
\Delta_{\max }=\frac{\varepsilon_{\max } E_{f}}{\sigma_{c}}
$$

and

$$
\Psi=\frac{\kappa h_{y} E_{f}}{\sigma_{c}}
$$

where $\varepsilon_{\max }$ is the tensile-face strain, $\kappa$ is the curvature, and $h_{y}$ is the beam thickness. 

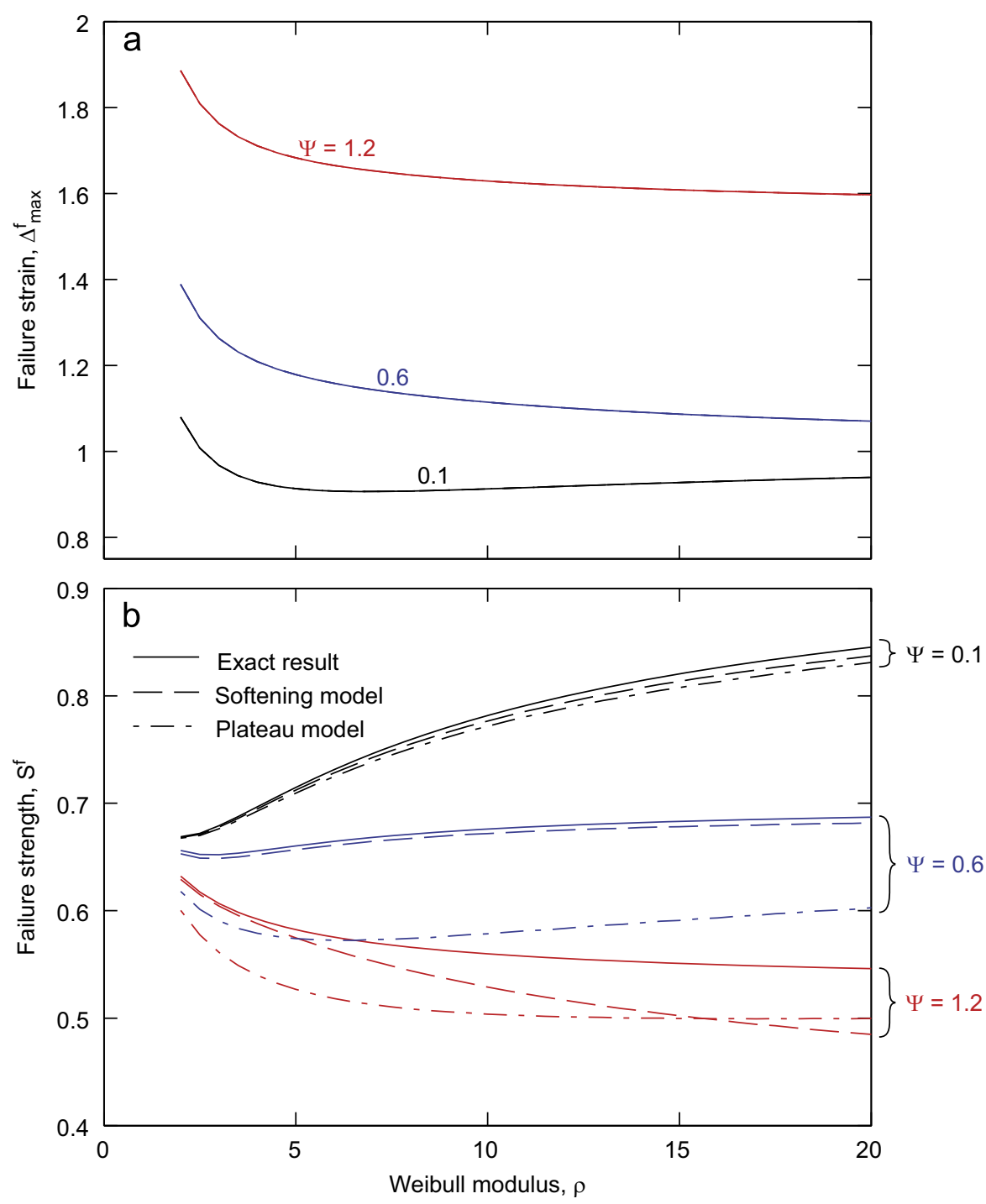

Fig. 7. Effects of Weibull modulus $\rho$ and strain gradient $\Psi$ on (a) failure strain and (b) failure strength (i.e. average hoop stress at failure) for the pressurized tube problem $\left(E^{\prime}=1 / 3\right.$ and $\left.S_{m c}=0\right)$.

Results for the limiting case of pure bending $(\lambda=\pi / 2)$ are plotted in Fig. 9. In contrast to the preceding model problems, bending failure occurs in one of two ways:

(i) At low $\rho$, a limit moment is obtained. Consequently, the tensile-face strain approaches infinity. Moreover, in the limit state, the stress throughout the beam is essentially uniform and equal to the plateau flow stress, $S_{p l}$ : the exception being the compressive face itself, where the stress approaches $-\infty$. In this domain the plateau model yields exact results; the limit stress is simply $S_{b}^{f}=3 S_{p l}$.

(ii) At higher values of $\rho$, a maximum moment is obtained. In this domain, failure occurs while the peak strain is on the softening portion of the stress-strain curve and thus the softening model yields highly accurate results. Furthermore, the failure stress is almost independent of $\rho$ and given to high accuracy by $S_{b}^{f}=C /\left(1+\sqrt{E^{\prime}}\right)$ with $C=1.92(<2 \%$ error for $\left.1 / 4<E^{\prime}<1, \rho \leq 20\right)^{1}$. Note that in contrast to the other two problems, the failure load in bending is somewhat sensitive to the elastic modulus, because much of the material is in compression. Similar results were obtained by Steif and Trojnacki (1994) using a tri-linear representation of the stress-strain curve. But, because of the absence of an explicit connection between the post-peak softening rate and the fiber properties as well as the absence of a plateau flow stress, their model is unable to predict the behavior at low $\rho$, especially the nature of the failure condition.

\footnotetext{
${ }^{1}$ The functional dependence of $S_{b}^{f}$ on $E^{\prime}$ was obtained using Curtin's approximate model. The parameter $C$ was then obtained by fitting this equation to the exact results from the Hui model.
} 


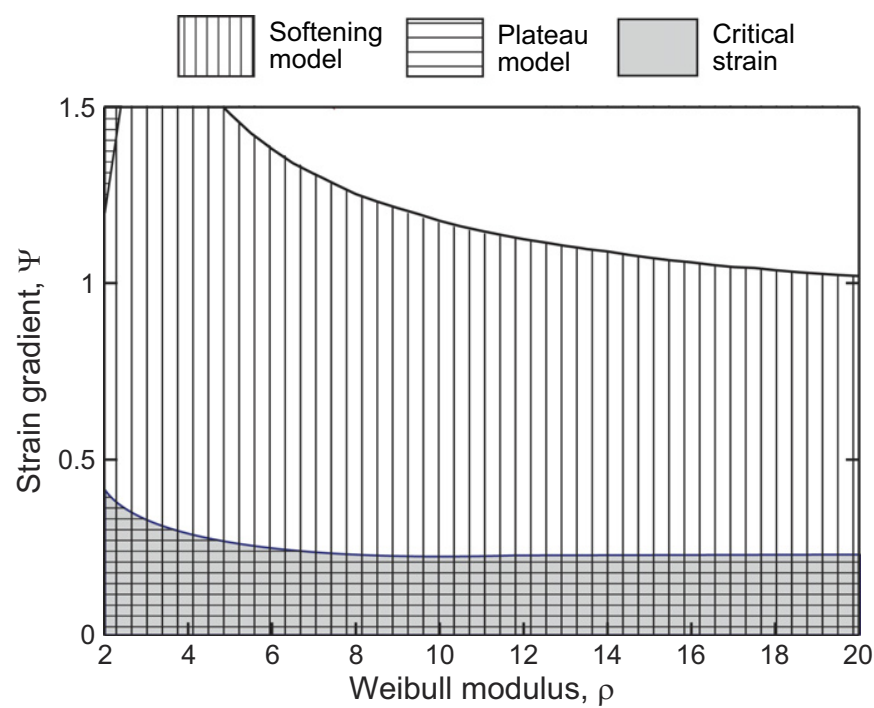

Fig. 8. Failure map for the pressurized tube problem $\left(E^{\prime}=1 / 3\right.$ and $\left.S_{m c}=0\right)$.

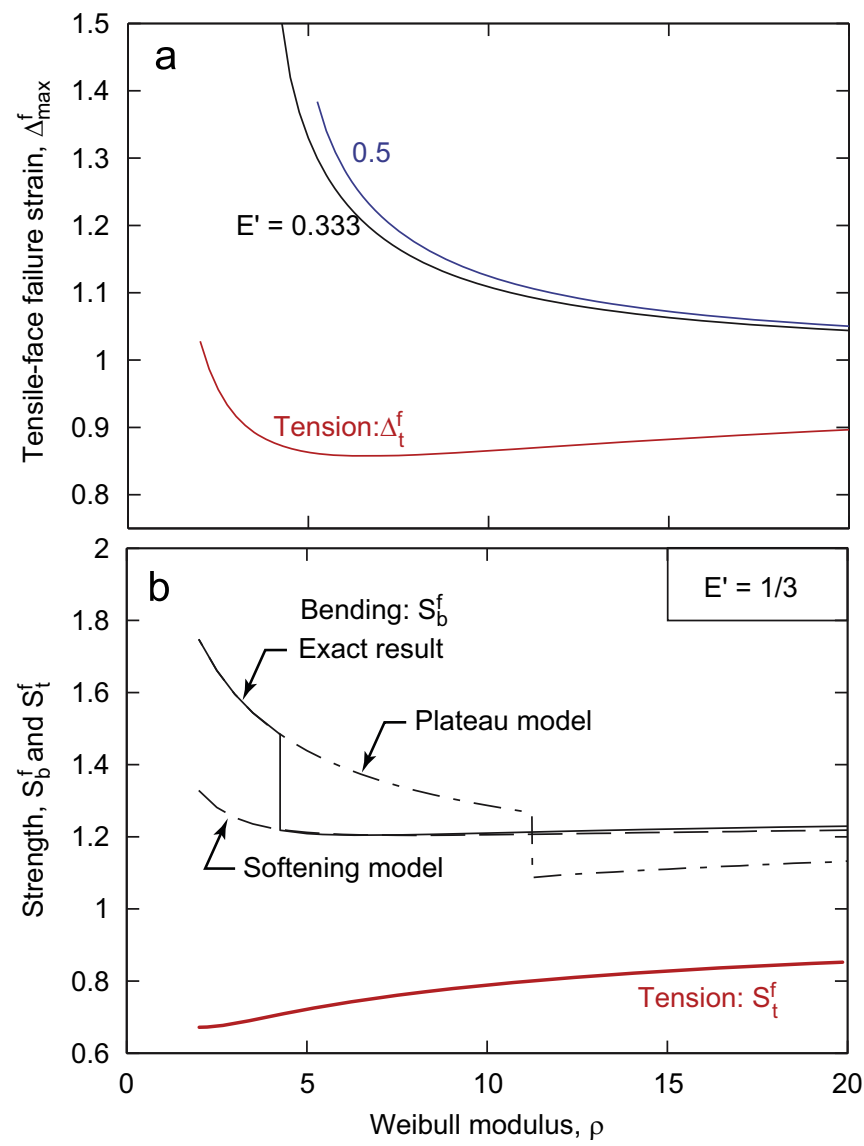

Fig. 9. (a) Failure strain and (b) strength in pure bending $\left(S_{m c}=0\right)$. Shown for comparison in red are the results for uniaxial tension.

The transition between the two domains is discontinuous and occurs at a critical value of $\rho$; for the case shown in Fig. 9, it occurs at $\rho \approx 4$. The plateau model also predicts a discontinuity, but not at the correct value of $\rho$. The softening model, on the other hand, does not predict the discontinuity, though it is accurate at high $\rho$. A key conclusion is that, despite the merits of the two approximate models in certain domains, neither satisfactorily predicts the behavior across the entire parameter range of interest. 
The failure loci for combined bending/tension are plotted in Fig. 10. The corresponding map showing the domains in which the approximate models yield adequate results is in Fig. 11. For the latter, the gradient is characterized by the tension/bending loading mixity parameter $\lambda$. The failure loci in $S_{t}^{f}-S_{b}^{f}$ space are approximately linear for fixed $\rho$. Indeed, for low $\rho$ and moderately high values of $\lambda$, wherein failure occurs via a limit condition, one can readily show using the plateau model that the failure locus is exactly linear and given by

$$
S_{t}^{f}+\frac{S_{b}^{f}}{3}=S_{p l}
$$

Interestingly, this result is independent of the peak stress in the fiber bundle response. But it breaks down at higher $\rho$ and lower $\lambda$, wherein failure occurs while the peak strain is in the softening regime, as evident in the map in Fig. 11. This behavior is seen in Fig. 10. For $\rho=3$, a discontinuity in the bending and tensile strengths occurs at low tension/bending mixity $\lambda$, since the failure condition transitions from a limit load to a load maximum.

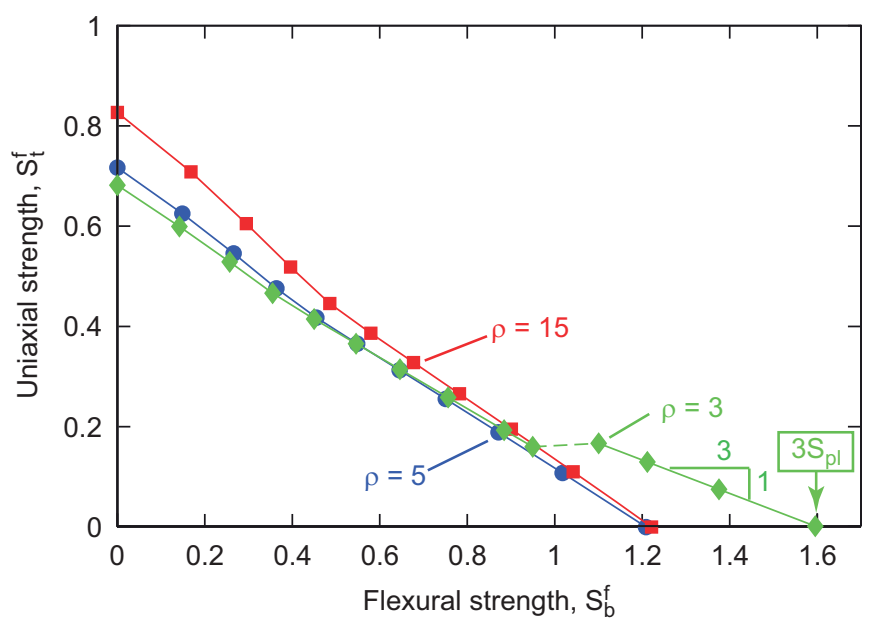

Fig. 10. Failure loci for combined bending and tension $\left(E^{\prime}=1 / 3\right.$ and $\left.S_{m c}=0\right)$.

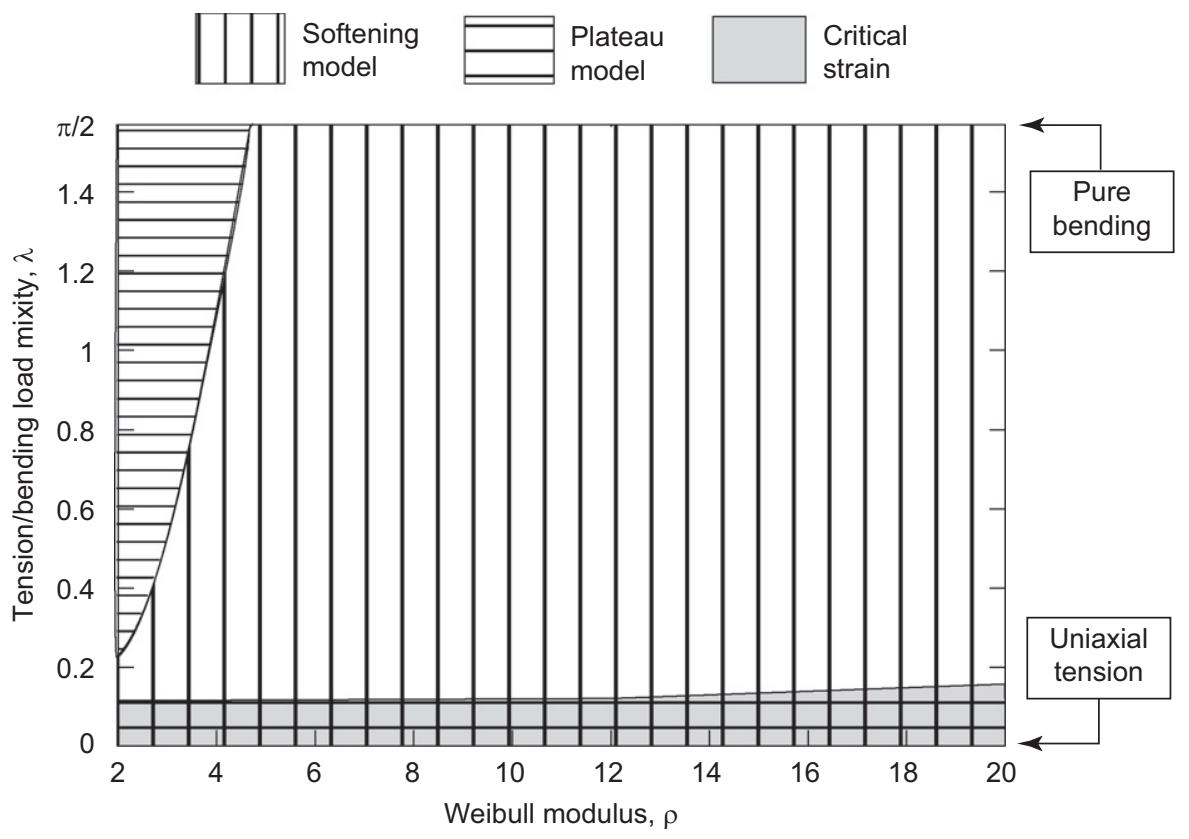

Fig. 11. Failure map for combined bending and tension $\left(E^{\prime}=1 / 3\right.$ and $\left.S_{m c}=0\right)$. 


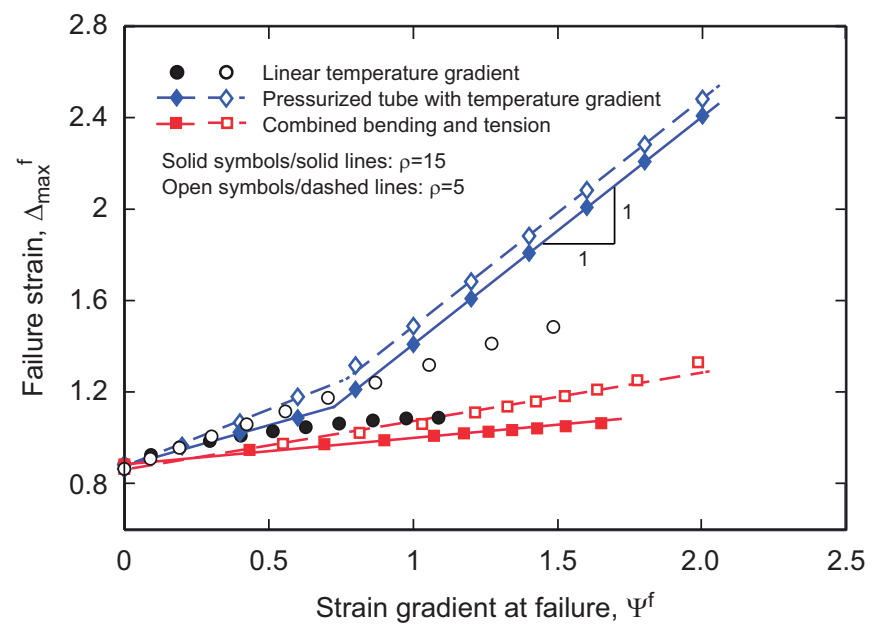

Fig. 12. Variation in the maximum strain at failure with the strain gradient at failure for the three model problems $\left(E^{\prime}=1 / 3\right.$ and $\left.S_{m c}=0\right)$.

\subsection{Comparisons of results from the three model problems}

Comparisons of the results from each of the three model problems are made on the basis of the variation in failure strain $\Delta_{\max }^{f}$ with the strain gradient $\Psi^{f}$ at failure (Fig. 12). Though the trends are qualitatively similar, there are significant differences in the details. This result is not unexpected, given the differences in loading trajectories. That is, in the linear temperature gradient problem, the strain gradient increases proportionally with strain; in the pressurized tube problem, the strain gradient is applied first and remains fixed while the pressure is increased; and for combined bending/tension, the loads are applied proportionally and thus the strain gradient is neither constant nor proportional to the maximum strain.

\section{Discussion}

A preliminary assessment of the present model has been made through comparisons with experimental measurements of failure stresses and strains under tensile and bending loads. Because of issues of delamination and/or compressive failure, we neglect the data on unidirectional CMCs and rely only on those for composites with multidirectional architectures and that are known to fail in a tensile mode under bending (McNulty and Zok, 1997). The data employed here are for a composite consisting of Nicalon fibers in a $\left[0^{\circ} / 90^{\circ}\right]$ layup and a magnesium aluminosilicate (MAS) matrix (Table 1). Both the tensile and compressive surfaces of the bend specimens had been instrumented with strain gauges. The ratios of bending/tension strengths and failure strains are 1.85-2.2 and 1.4-1.6, respectively. For the model predictions, we select a range of values of $E^{\prime}$ and $S_{m c}$ that realistically match those obtained from the measured tensile stress-strain curves $\left(E^{\prime}=0.17-0.25, S_{m c}=0.3-0.4\right)$. Furthermore, we assume a fiber Weibull modulus $\rho=4$ (typical of Nicalon fibers (McNulty and Zok, 1997)). The predicted ratios of bending/tension strength and failure strain are 1.85-2 and 1.5-1.6, respectively. The predictions agree quite well with the experimental results. By comparison, using the same parameter values along with a critical strain criterion for fracture, the predicted strength and failure strain ratios are $1.5-1.7$ and 1 , respectively. The latter results indicate that neglecting the contribution from the post-peak regime results in the flexural strength being underestimated by approximately $20 \%$. The ratio of failure strains is (naturally) underestimated by an even larger margin. We further note that other mechanics-based models for composite fracture also fail to accurately predict the ratio of failure strains (Neumeister, 1993b; Hild et al., 1994). Additional critical experiments to probe effects of gradients on failure are in progress and will be reported elsewhere.

As noted at the outset, the present analysis is predicated on the assumption of global load sharing (GLS) among fibers: a condition obtained when the interface toughness and sliding stress are low. Otherwise, some degree of stress concentration is obtained around a fiber failure site (Hedgepeth and van Dyke, 1967). The effects of local load sharing (LLS) on fiber bundle properties have been studied by Curtin and co-workers (Ibnabdeljalil and Curtin, 1997; Zhou and Curtin, 1995) using threedimensional lattice spring models. Their results show that, for spring constants that yield realistic values of stress concentrations around fiber breaks, the fiber bundle strength is reduced only slightly relative to the GLS prediction and exhibits only a small amount of variability and volume-sensitivity. Furthermore, the bundle properties follow the same scalings with the characteristic strength $\sigma_{c}$ and the characteristic length $\delta_{c}$. The inference is that, although the present work has been based on the GLS assumption, the results for LLS conditions are likely to be similar. Furthermore, the ratios of quantities, such as flexural/tensile strengths, should be predicted with reasonable accuracy.

Finally, we return to the issue of deformation localization once the stress locally reaches the peak in the stress-strain curve. As argued earlier, if a localization band were to spread under an increasing macroscopic load, then other potential localization sites remote from the first could be triggered. Averaging the displacements over an appropriate length scale 


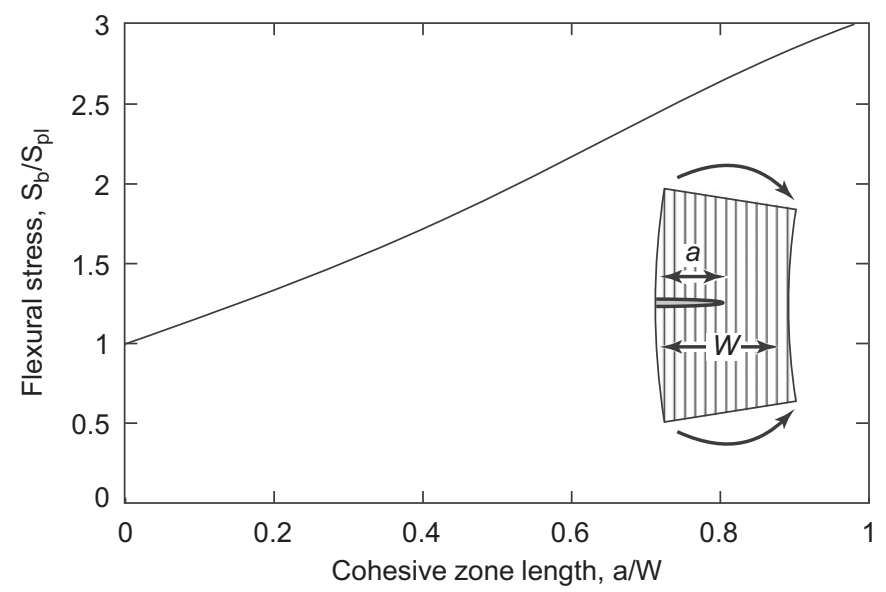

Fig. 13. Flexural stress corresponding to the growth of a localized deformation band through a bend specimen.

would yield a physically-meaningful strain. Indeed, this situation is the same as that of a matrix crack in a CMC bridged by fully-intact fibers under uniaxial tensile loading. In the latter scenario, the deformation is clearly localized in the vicinity of the crack (manifested as a crack opening displacement). But, because of structural hardening (in this case resulting from a rising traction law), other cracks form in an approximately periodic manner, ultimately reaching a spacing dictated by the slip length along the fiber-matrix interface; the displacements are averaged accordingly to yield a macroscopic inelastic strain. There is no reason to believe that the same macroscopic response - with multiple periodic cracks - should not be obtained in cases where the traction law associated with a crack exhibits a softening portion provided the loading is such that crack extension occurs under an increasing macroscopic load. The expectation therefore is that a cohesive approach should yield a peak macroscopic load that is virtually identical to that obtained from a continuum description of the inelastic deformation.

The latter equivalence can be demonstrated using a specific example. We consider the problem of pure bending of a composite with a low fiber Weibull modulus and hence a high plateau flow stress. The results in Section 3.4 show that, in this scenario, a limit moment is obtained, with a predicted bending strength of $S_{b}^{f}=3 S_{p l}$. Arguably the problem could be tackled equivalently by considering the extension of a single localized deformation band from the tensile face towards the compressive face, with a bridging stress equal to $S_{p l}$. The growth of the band can be readily computed using a standard cohesive zone approach. ${ }^{2}$ The corresponding fracture resistance curve, couched in terms of the macroscopic bending stress and the current length of the localized band, is plotted in Fig. 13. The band grows stably from a stress of $S_{p l}$ at its inception to a maximum value of $3 S_{p l}$ when it reaches the back face: the latter being identical to that predicted from the limit moment calculation. Despite the utility of the cohesive zone model in predicting the ultimate bending strength of such a composite, the model provides no information about the density of localized bands and the associated macroscopic strain. This aspect of the problem would require consideration of the pull-out lengths of fiber fragments and the interactions between adjacent localized bands. The result is likely to be very similar to that obtained from Hui's fragmentation model.

\section{Conclusions}

In this paper, we have employed an exact model of fiber fragmentation to predict failure of CMCs in non-uniform strain fields. A key distinction from approaches adopted by others is in the use of a failure criterion based on the attainment of a load maximum. Assessments based on comparisons with the few reliable experimental data available in the literature on bending and tensile properties of CMCs are encouraging. In particular, the model is able to predict rather well the failure strain ratio: a feature not captured by previous models, especially those based on a critical-strain failure criterion.

Approximations to the exact fragmentation model have also been assessed. The approximations were selected to decouple the effects of the post-peak strain softening and the plateau "flow" stress at high strains. While neither of the two approximate representations yields accurate results for strengths over the entire parameter space of interest, each exhibits merits over limited domains. For instance, in cases in which the fiber Weibull modulus is low and the loading yields a sufficiently high strain gradient, the failure strain lies well within the plateau regime. Consequently, strengths can be predicted with good accuracy using a perfectly-plastic representation of the post-peak response. Indeed, in the limiting case of pure bending, the latter model yields exact results for low values of Weibull modulus. In contrast, for cases in which the fiber Weibull modulus is high, the failure strain lies in the softening portion of the curve. Here a linear-softening

\footnotetext{
2 The results were computed using the analytical formulae for weight functions on pages 2.13 and 2.27 of Tada et al. (1985), assuming zero crack tip toughness. Note that, since the bridging stress is constant, there is no additional (bridging) length scale in the problem. A corollary to this statement is the tacit assumption that the peak crack opening displacement remains small in relation to the characteristic fiber pull-out length.
} 
model is found to yield accurate results. In yet other (rarer) circumstances, the salient features of fracture cannot be captured by either one of the two approximations and thus the full fragmentation model is required for accurate strength predictions.

\section{Acknowledgements}

This work was supported by the Pratt and Whitney Center of Excellence at the University of California, Santa Barbara (monitored by Douglas Berczik) as well as the US AFOSR (Dr. Ali Sayir) and NASA (Dr. Anthony Calomino) under the National Hypersonics Science Center for Materials and Structures. VPR was supported in part by a National Defense Science and Engineering Graduate Fellowship.

\section{Appendix A. Exact fragmentation model}

Here we present the exact analytical descriptions of the fragmentation model developed by Hui et al (1995). The average fiber stress is partitioned into three terms:

$$
S(\Delta)=\int_{0}^{\Delta / 2} \frac{\ell^{2}}{2} p_{1}(\Delta, \ell) d x+\int_{\Delta / 2}^{\Delta} \frac{\ell^{2}}{2} p_{2}(\Delta, \ell) d \ell+\int_{\Delta}^{\infty}\left[(\ell-\Delta) \Delta+\frac{\Delta^{2}}{2}\right] p_{3}(\Delta, \ell) \mathrm{d} \ell
$$

The three integrals on the right side correspond to contributions from fragments of length $\ell<\Delta / 2, \Delta / 2<\ell<\Delta$, and $\ell>\Delta$, respectively. The fragment lengths are normalized by the characteristic length, $\delta_{c}$. The functions $p_{i}(\Delta, \ell)$ represent fiber fragment densities, given by

$$
\begin{aligned}
& p_{1}(\Delta, \ell)=p(\ell)+2 \rho \int_{\ell}^{2 \ell} \frac{A_{0}(t)}{t} \exp \left[-t^{p}\left(\ell+\frac{t}{2}\right)\right] d t \\
& p_{2}(\Delta, \ell)=p(\ell)+2 \rho \int_{\ell}^{\Delta} \frac{A_{0}(t)}{t} \exp \left[-t^{p}\left(\ell+\frac{t}{2}\right)\right] d t \\
& p_{3}(\Delta, \ell)=A_{0}(\Delta) \exp \left(-\Delta^{\rho} \ell\right)
\end{aligned}
$$

where

$$
\begin{aligned}
& p(\ell)=\ell^{2 \rho} \exp \left[\frac{-\ell^{\rho+1}}{\rho+1}\right] \psi\left(\frac{\ell^{\rho+1}}{2}\right) \\
& A_{0}(\ell)=\ell^{2 \rho} \exp \left[\frac{\rho \ell^{\rho+1}}{\rho+1}\right] \psi\left(\frac{\ell^{\rho+1}}{2}\right)
\end{aligned}
$$

and

$$
\psi(x)=\exp \left(\frac{-2 \rho}{\rho+1} \int_{0}^{x} \frac{1-\exp (-t)}{t} d t\right)
$$

\section{References}

Aveston, J., Cooper, G.A., Kelly, A., 1971. Single and multiple fracture, in: The Properties of Fiber Composites, National Physical Laboratory. IPC Science and Technology Press Ltd. pp. 15-24.

Beyerle, D.S., Spearing, S.M., Zok, F.W., Evans, A.G., 1992. Damage and failure in unidirectional ceramic-matrix composites. J. Am. Ceram. Soc. 75, 2719-2725.

Bullock, R.E., 1974. Strength ratios of composite materials in flexure and in tension. J. Comp. Mater. 8, 200-206.

Cady, C., Heredia, F.E., Evans, A.G., 1995. In-plane mechanical properties of several ceramic-matrix composites. J. Am. Ceram. Soc. 78, $2065-2078$.

Cox, H.L., 1952. The elasticity and strength of paper and other fibrous materials. Br. J. App. Phys. 3, 72-79.

Curtin, W.A., 1991a. Exact theory of fibre fragmentation in a single-filament composite. J. Mater. Sci. 26, 5239-5253.

Curtin, W.A., 1991b. Theory of mechanical properties of ceramic-matrix composites. J. Am. Ceram. Soc. 74, 2837-2845.

Curtin, W.A., 1998. Stochastic damage evolution and failure in fiber-reinforced composites. Volume 36 of Advances in Applied Mechanics. Elsevier pages $163-253$.

Curtin, W.A., Ahn, B.K., Takeda, N., 1998. Modeling brittle and tough stress-strain behavior in unidirectional ceramic matrix composites. Acta Mater. 46, 3409-3420.

Evans, A.G., Zok, F.W., 1994. The physics and mechanics of fibre-reinforced brittle matrix composites. J. Mater. Sci. 29, $3857-3896$.

Hedgepeth, J.M., van Dyke, P., 1967. Local stress concentrations in imperfect filamentary composite materials. J. Comp. Mater. 1, 294-309. 
Hild, F., Domergue, J.M., Leckie, F.A., Evans, A.G., 1994. Tensile and flexural ultimate strength of fiber-reinforced ceramic-matrix composites. Int. J. Solids Struct. 31, 1035-1045.

Hui, C.Y., Phoenix, S.L., Ibnabdeljalil, M., Smith, R.L., 1995. An exact closed form solution for fragmentation of weibull fibers in a single filament composite with applications to fiber-reinforced ceramics. J. Mech. Phys. Solids 43, 1551-1585.

Ibnabdeljalil, M., Curtin, W.A., 1997. Strength and reliability of fiber-reinforced composites: localized load-sharing and associated size effects. Int. J. Solids Struct. 34, 2649-2668.

Jansson, S., Leckie, F.A., 1992. The mechanics of failure of silicon carbide fiber reinforced glass-matrix composites. Acta Metall. Mater. 40, $2967-2978$.

Marshall, D.B., Evans, A.G., 1985. Failure mechanisms in ceramic-fiber/ceramic matrix composites. J. Am. Ceram. Soc. 68, $225-231$.

MATLAB Version 7.9.0.529 (R2009b), 2009. The MathWorks, Inc., Natick, Massachusetts.

McNulty, J.C., Zok, F.W., 1997. Application of weakest-link fracture statistics to fiber-reinforced ceramic-matrix composites. J. Am. Ceram. Soc. 80 , 1535-1543.

McNulty, J.C., Zok, F.W., Genin, G.M., Evans, A.G., 1999. Notch-sensitivity of fiber-reinforced ceramic-matrix composites: effects of inelastic straining and volume-dependent strength. J. Am. Ceram. Soc. 82, 1217-1228.

Neumeister, J.M., 1993a. A constitutive law for continuous fiber reinforced brittle matrix composites with fiber fragmentation and stress recovery. J. Mech. Phys. Solids 41, 1383-1404.

Neumeister, J.M., 1993b. Bundle pullout: a failure mechanism limiting the tensile strength of continuous fiber reinforced brittle matrix composites and its implications for strength dependence on volume and type of loading. J. Mech. Phys. Solids 41, 1405-1424.

Phoenix, S.L., 1993. Statistical issues in the fracture of brittle-matrix fibrous composites. Comp. Sci. Tech. 48, 65-80.

Prewo, K.M., 1986. Tension and flexural strength of silicon carbide fiber-reinforced glass ceramics. J. Mater. Sci. 21, 3590-3600.

Ramamurty, U., 2004. Assessment of load transfer characteristics of a fiber-reinforced titanium-matrix composite. Comp. Sci. Tech. 65, $1815-1825$.

Steif, P.S., Trojnacki, A., 1994. Bend strength vs. tensile strength of fiber-reinforced ceramics. J. Am. Ceram. Soc. 77, 221-229.

Tada, H., Paris, P.C., Irwin, G.R., 1985. The stress analysis of cracks handbook, 2nd edition Paris Productions Inc., St. Louis, Missouri.

Thouless, M.D., Evans, A.G., 1988. Effects of pull-out on the mechanical properties of ceramic-matrix composites. Acta Metall. 36, 517-522.

Whitney, J.M., Knight, M., 1980. The relationship between tensile strength and flexure strength in fiber-reinforced composites. Exp. Mech. 20, 211-216.

Zhou, S.J., Curtin, W.A., 1995. Failure of fiber composites: a lattice Green function model. Acta Metall. Mater. 43, 3094-3104. 